\title{
Initial Coefficient estimates for a classes of $m$-fold symmetric bi-univalent functions involving Mittag-Leffler function
}

\author{
Abbas Kareem Wanas, Huo Tang
}

\begin{abstract}
The main object of the present paper is to use MittagLeffler function to introduce and study two new classes

$$
\mathcal{R}_{\Sigma_{m}}(\gamma, \lambda, \eta, \delta, \tau ; \alpha) \quad \text { and } \quad \mathcal{R}_{\Sigma_{m}}^{*}(\gamma, \lambda, \eta, \delta, \tau ; \beta)
$$

of $\Sigma_{m}$ consisting of analytic and $m$-fold symmetric bi-univalent functions defined in the open unit disk $U$. Also, we determine the estimates on the initial coefficients $\left|a_{m+1}\right|$ and $\left|a_{2 m+1}\right|$ for functions in each of these new classes. Furthermore, we indicate certain special cases for our results.
\end{abstract}

\section{INTRODUCTION}

Let $\mathcal{A}$ stands for the class of functions $f$ that are analytic in the open unit disk $U=\{z \in \mathbb{C}:|z|<1\}$, are normalized by the conditions $f(0)=$ $f^{\prime}(0)-1=0$, and have the form:

$$
f(z)=z+\sum_{k=2}^{\infty} a_{k} z^{k} .
$$

Let $S$ be the subclass of $\mathcal{A}$ consisting of functions of the form (1) which are also univalent in $U$. The Koebe one-quarter theorem (see [5]) states that the image of $U$ under every function $f \in S$ contains a disk of radius $\frac{1}{4}$. Therefore, every function $f \in S$ has an inverse $f^{-1}$ which satisfies $f^{-1}(f(z))=z$, $(z \in U)$ and $f\left(f^{-1}(w)\right)=w,\left(|w|<r_{0}(f), r_{0}(f) \geq \frac{1}{4}\right)$, where

$$
\begin{aligned}
g(w)=f^{-1}(w)= & w-a_{2} w^{2}+\left(2 a_{2}^{2}-a_{3}\right) w^{3} \\
& -\left(5 a_{2}^{3}-5 a_{2} a_{3}+a_{4}\right) w^{4}+\cdots .
\end{aligned}
$$

A function $f \in \mathcal{A}$ is said to be bi-univalent in $U$ if both $f$ and $f^{-1}$ are univalent in $U$. We denote by $\Sigma$ the class of bi-univalent functions in $U$

2010 Mathematics Subject Classification. Primary: 30C45; Secondary: 30C50.

Key words and phrases. Analytic function, $m$-fold symmetric bi-univalent function, Coefficient bounds, Mittag-Leffler function.

Full paper. Received 9 March 2020, revised 4 June 2020, accepted 6 June 2020, available online 15 June 2020. 
satisfying (1). In fact, Srivastava et al. [18] have actually revived the study of analytic and bi-univalent functions in recent years, it was followed by such works as those by Murugusundaramoorthy et al. [11], Caglar et al. [4], Adegani et al. [1] and others (see, for example [8, 13, 14, 17, 25]).

For each function $f \in S$, the function $h(z)=\left(f\left(z^{m}\right)\right)^{\frac{1}{m}},(z \in U, m \in \mathbb{N})$ is univalent and maps the unit disk $U$ into a region with $m$-fold symmetry. A function is said to be $m$-fold symmetric (see [7]) if it has the following normalized form:

$$
f(z)=z+\sum_{k=1}^{\infty} a_{m k+1} z^{m k+1}, \quad(z \in U, m \in \mathbb{N}) .
$$

We denote by $S_{m}$ the class of $m$-fold symmetric univalent functions in $U$, which are normalized by the series expansion (3). In fact, the functions in the class $S$ are one-fold symmetric.

In [20] Srivastava et al. defined $m$-fold symmetric bi-univalent functions analogues to the concept of $m$-fold symmetric univalent functions. They gave some important results, such as each function $f \in \Sigma$ generates an $m$ fold symmetric bi-univalent function for each $m \in \mathbb{N}$. Furthermore, for the normalized form of $f$ given by (3), they obtained the series expansion for $f^{-1}$ as follows:

$$
\begin{aligned}
& g(w)=w-a_{m+1} w^{m+1}+\left[(m+1) a_{m+1}^{2}-a_{2 m+1}\right] w^{2 m+1} \\
& -\left[\frac{1}{2}(m+1)(3 m+2) a_{m+1}^{3}-(3 m+2) a_{m+1} a_{2 m+1}+a_{3 m+1}\right] w^{3 m+1}+\cdots,
\end{aligned}
$$

where $f^{-1}=g$. We denote by $\Sigma_{m}$ the class of $m$-fold symmetric bi-univalent functions in $U$. It is easily seen that for $m=1$, the formula (4) coincides with the formula (2) of the class $\Sigma$. Some examples of $m$-fold symmetric bi-univalent functions are given as follows:

$$
\left(\frac{z^{m}}{1-z^{m}}\right)^{\frac{1}{m}}, \quad\left[\frac{1}{2} \log \left(\frac{1+z^{m}}{1-z^{m}}\right)\right]^{\frac{1}{m}}, \quad\left[-\log \left(1-z^{m}\right)\right]^{\frac{1}{m}}
$$

with the corresponding inverse functions

$$
\left(\frac{w^{m}}{1+w^{m}}\right)^{\frac{1}{m}},\left(\frac{e^{2 w^{m}}-1}{e^{2 w^{m}}+1}\right)^{\frac{1}{m}}\left(\frac{e^{w^{m}}-1}{e^{w^{m}}}\right)^{\frac{1}{m}},
$$

respectively.

Recently, many authors investigated bounds for various subclasses of $m$ fold bi-univalent functions (see $[2,6,12,15,16,19,22,23,24,26,27]$ ).

The Mittag-Leffler function $E_{\lambda}(z),(z \in \mathbb{C})$ (see $[9,10]$ ) is defined by

$$
E_{\lambda}(z)=\sum_{k=0}^{\infty} \frac{z^{k}}{\Gamma(\lambda k+1)}, \quad(\lambda \in \mathbb{C}, \operatorname{Re}(\lambda)>0) .
$$


Recently, Srivastava and Tomovski [21] introduced the function $E_{\lambda, \eta}^{\delta, \tau}(z)$, $(z \in \mathbb{C})$ in the form:

$$
E_{\lambda, \eta}^{\delta, \tau}(z)=\sum_{k=0}^{\infty} \frac{(\delta)_{k \tau} z^{k}}{\Gamma(\lambda k+\eta) k !}, \quad(\lambda \in \mathbb{C}, \operatorname{Re}(\lambda)>0) .
$$

where $\lambda, \eta, \delta \in \mathbb{C}, \operatorname{Re}(\lambda)>\max \{0, \operatorname{Re}(\tau)-1\}, \operatorname{Re}(\tau)>0$ and $(x)_{k}$ is the Pochhammer symbol defined by

$$
(x)_{k}=\frac{\Gamma(x+k)}{\Gamma(x)}=\left\{\begin{array}{cc}
1, & \text { for } k=0 \\
x(x+1) \cdots(x+n-1), & \text { for } k \in \mathbb{N}
\end{array}\right.
$$

In 2016, Attiya [3] introduced and investigated a linear operator $\mathcal{H}_{\lambda, \eta}^{\delta, \tau}$ : $\mathcal{A} \longrightarrow \mathcal{A}$ by using the Hadamard product (or convolution) and defined as follows

$$
\mathcal{H}_{\lambda, \eta}^{\delta, \tau} f(z)=Q_{\lambda, \eta}^{\delta, \tau}(z) * f(z), \quad(z \in U),
$$

where "*" indicate the Hadamard product (or convolution) of two series and

$$
Q_{\lambda, \eta}^{\delta, \tau}(z)=\frac{\Gamma(\lambda+\eta)}{(\delta)_{\tau}}\left(E_{\lambda, \eta}^{\delta, \tau}(z)-\frac{1}{\Gamma(\eta)}\right)
$$

$\lambda, \eta, \delta \in \mathbb{C}, \operatorname{Re}(\lambda)>\max \{0, \operatorname{Re}(\tau)-1\}, \operatorname{Re}(\tau)>0$.

By some easy computations, we conclude that

$$
\mathcal{H}_{\lambda, \eta}^{\delta, \tau} f(z)=z+\sum_{k=2}^{\infty} \frac{\Gamma(\delta+k \tau) \Gamma(\lambda+\eta)}{\Gamma(\delta+\tau) \Gamma(\lambda k+\eta) \Gamma(k+1)} a_{k} z^{k} .
$$

It is easily verified that if $f \in S_{m}$, then we have

$$
\mathcal{H}_{\lambda, \eta}^{\delta, \tau} f(z)=z+\sum_{k=1}^{\infty} \frac{\Gamma(\delta+(m k+1) \tau) \Gamma(\lambda+\eta)}{\Gamma(\delta+\tau) \Gamma(\lambda(m k+1)+\eta) \Gamma(m k+2)} a_{m k+1} z^{m k+1} .
$$

We require the following lemma to prove our main results.

Lemma 1. [5] If $h \in \mathcal{P}$, then $\left|c_{k}\right| \leq 2$ for each $k \in \mathbb{N}$, where $\mathcal{P}$ is the family of all functions $h$ analytic in $U$ for which

$$
\operatorname{Re}(h(z))>0, \quad(z \in U),
$$

where

$$
h(z)=1+c_{1} z+c_{2} z^{2}+\cdots, \quad(z \in U) .
$$

2. Coefficient estimates For the FUnCtion

$$
\text { CLASS } \mathcal{R}_{\Sigma_{m}}(\gamma, \lambda, \eta, \delta, \tau ; \alpha)
$$

Definition 1. For $0<\alpha \leq 1,0 \leq \gamma \leq 1$ and $m \in \mathbb{N}$, a function $f \in \Sigma_{m}$ given by (3) is said to be in the class $\mathcal{R}_{\Sigma_{m}}(\gamma, \lambda, \eta, \delta, \tau ; \alpha)$, if it satisfies the following conditions:

$$
\left|\arg \left(\gamma z\left(\mathcal{H}_{\lambda, \eta}^{\delta, \tau} f(z)\right)^{\prime \prime}+(2 \gamma+1)\left(\mathcal{H}_{\lambda, \eta}^{\delta, \tau} f(z)\right)^{\prime}-2 \gamma\right)\right|<\frac{\alpha \pi}{2},
$$


and

$$
\left|\arg \left(\gamma w\left(\mathcal{H}_{\lambda, \eta}^{\delta, \tau} g(w)\right)^{\prime \prime}+(2 \gamma+1)\left(\mathcal{H}_{\lambda, \eta}^{\delta, \tau} g(w)\right)^{\prime}-2 \gamma\right)\right|<\frac{\alpha \pi}{2},
$$

where the function $g=f^{-1}$ is given by (4).

In particular, for one-fold symmetric bi-univalent functions, we denote the class

$$
\mathcal{R}_{\Sigma_{1}}(\gamma, \lambda, \eta, \delta, \tau ; \alpha) \equiv \mathcal{R}_{\Sigma}(\gamma, \lambda, \eta, \delta, \tau ; \alpha)
$$

Remark 1. If we put $\gamma=\lambda=0$ and $\eta=\delta=\tau=1$ :

(1) The class $\mathcal{R}_{\Sigma_{m}}(\gamma, \lambda, \eta, \delta, \tau ; \alpha)$ reduces to the class $\mathcal{H}_{\Sigma, m}^{\alpha}$ which was given by Srivastava et al. [20];

(2) The class $\mathcal{R}_{\Sigma}(\gamma, \lambda, \eta, \delta, \tau ; \alpha)$ reduces to the class $\mathcal{H}_{\Sigma}^{\alpha}$ which was given by Srivastava et al. [18].

Theorem 1. Let $f \in \mathcal{R}_{\Sigma_{m}}(\gamma, \lambda, \eta, \delta, \tau ; \alpha)(0<\alpha \leq 1,0 \leq \gamma \leq 1, m \in \mathbb{N})$ be given by (3). Then

$$
\begin{aligned}
& \left|a_{m+1}\right| \leq \\
& \frac{2 \alpha \Gamma(\delta+\tau) \Gamma(\lambda(m+1)+\eta) \Gamma(m+2) \sqrt{\Gamma(\lambda(2 m+1)+\eta) \Gamma(2(m+1))}}{\sqrt{\left|\alpha \Omega_{1}(\gamma, \lambda, \eta, \delta, \tau, m)+(1-\alpha) \Gamma(\lambda(2 m+1)+\eta) \Gamma(2(m+1)) \Omega_{2}^{2}(\gamma, \lambda, \eta, \delta, \tau, m)\right|}}
\end{aligned}
$$

and

$$
\begin{aligned}
& \left|a_{2 m+1}\right| \leq \frac{2 \alpha^{2}(m+1) \Gamma^{2}(\delta+\tau) \Gamma^{2}(\lambda(m+1)+\eta) \Gamma^{2}(m+2)}{\Omega_{2}^{2}(\gamma, \lambda, \eta, \delta, \tau, m)} \\
& \quad+\frac{2 \alpha \Gamma(\delta+\tau) \Gamma(\lambda(2 m+1)+\eta) \Gamma(2(m+1))}{\Gamma(\delta+(2 m+1) \tau) \Gamma(\lambda+\eta)[\gamma(4 m(m+1)+2)+2 m(\gamma+1)+1]}
\end{aligned}
$$

where

$$
\begin{aligned}
\Omega_{1}(\gamma, \lambda, \eta, \delta, \tau, m)= & (m+1) \Gamma(\delta+\tau) \Gamma^{2}(\lambda(m+1)+\eta) \times \\
& \times \Gamma^{2}(m+2) \Gamma(\delta+(2 m+1) \tau) \Gamma(\lambda+\eta) \times \\
& \times[\gamma(4 m(m+1)+2)+2 m(\gamma+1)+1], \\
\Omega_{2}(\gamma, \lambda, \eta, \delta, \tau, m)= & \Gamma(\delta+(m+1) \tau) \Gamma(\lambda+\eta) \times \\
& \times\left[\gamma\left((m+1)^{2}+1\right)+m(\gamma+1)+1\right] .
\end{aligned}
$$

Proof. It follows from conditions (5) and (6) that

$$
\gamma z\left(\mathcal{H}_{\lambda, \eta}^{\delta, \tau} f(z)\right)^{\prime \prime}+(2 \gamma+1)\left(\mathcal{H}_{\lambda, \eta}^{\delta, \tau} f(z)\right)^{\prime}-2 \gamma=[p(z)]^{\alpha}
$$

and

$$
\gamma w\left(\mathcal{H}_{\lambda, \eta}^{\delta, \tau} g(w)\right)^{\prime \prime}+(2 \gamma+1)\left(\mathcal{H}_{\lambda, \eta}^{\delta, \tau} g(w)\right)^{\prime}-2 \gamma=[q(w)]^{\alpha},
$$

where $g=f^{-1}$ and $p, q$ in $\mathcal{P}$ have the following series representations:

$$
p(z)=1+p_{m} z^{m}+p_{2 m} z^{2 m}+p_{3 m} z^{3 m}+\cdots
$$


and

$$
q(w)=1+q_{m} w^{m}+q_{2 m} w^{2 m}+q_{3 m} w^{3 m}+\cdots .
$$

Comparing the corresponding coefficients of (11) and (12) yields

$$
\begin{aligned}
& \frac{\Gamma(\delta+(m+1) \tau) \Gamma(\lambda+\eta)\left[\gamma\left((m+1)^{2}+1\right)+m(\gamma+1)+1\right]}{\Gamma(\delta+\tau) \Gamma(\lambda(m+1)+\eta) \Gamma(m+2)} a_{m+1}=\alpha p_{m}, \\
& \frac{\Gamma(\delta+(2 m+1) \tau) \Gamma(\lambda+\eta)[\gamma(4 m(m+1)+2)+2 m(\gamma+1)+1]}{\Gamma(\delta+\tau) \Gamma(\lambda(2 m+1)+\eta) \Gamma(2(m+1))} a_{2 m+1} \\
& \quad=\alpha p_{2 m}+\frac{\alpha(\alpha-1)}{2} p_{m}^{2},
\end{aligned}
$$

$$
-\frac{\Gamma(\delta+(m+1) \tau) \Gamma(\lambda+\eta)\left[\gamma\left((m+1)^{2}+1\right)+m(\gamma+1)+1\right]}{\Gamma(\delta+\tau) \Gamma(\lambda(m+1)+\eta) \Gamma(m+2)} a_{m+1}=\alpha q_{m}
$$

and

$$
\begin{aligned}
& \frac{\Gamma(\delta+(2 m+1) \tau) \Gamma(\lambda+\eta)[\gamma(4 m(m+1)+2)+2 m(\gamma+1)+1]}{\Gamma(\delta+\tau) \Gamma(\lambda(2 m+1)+\eta) \Gamma(2(m+1))} \times \\
& \times\left((m+1) a_{m+1}^{2}-a_{2 m+1}\right)=\alpha q_{2 m}+\frac{\alpha(\alpha-1)}{2} q_{m}^{2} .
\end{aligned}
$$

In view of (15) and (17), we find that

$$
p_{m}=-q_{m}
$$

and

$$
\begin{aligned}
& \frac{2 \Gamma^{2}(\delta+(m+1) \tau) \Gamma^{2}(\lambda+\eta)\left[\gamma\left((m+1)^{2}+1\right)+m(\gamma+1)+1\right]^{2}}{\Gamma^{2}(\delta+\tau) \Gamma^{2}(\lambda(m+1)+\eta) \Gamma^{2}(m+2)} a_{m+1}^{2} \\
& =\alpha^{2}\left(p_{m}^{2}+q_{m}^{2}\right) .
\end{aligned}
$$

Also, from (16), (18) and (20), we obtain

$$
\begin{aligned}
& \frac{(m+1) \Gamma(\delta+(2 m+1) \tau) \Gamma(\lambda+\eta)[\gamma(4 m(m+1)+2)+2 m(\gamma+1)+1]}{\Gamma(\delta+\tau) \Gamma(\lambda(2 m+1)+\eta) \Gamma(2(m+1))} a_{m+1}^{2} \\
& =\alpha\left(p_{2 m}+q_{2 m}\right)+\frac{\alpha(\alpha-1)}{2}\left(p_{m}^{2}+q_{m}^{2}\right)=\alpha\left(p_{2 m}+q_{2 m}\right)+ \\
& +\frac{(\alpha-1) \Gamma^{2}(\delta+(m+1) \tau) \Gamma^{2}(\lambda+\eta)\left[\gamma\left((m+1)^{2}+1\right)+m(\gamma+1)+1\right]^{2}}{\alpha \Gamma^{2}(\delta+\tau) \Gamma^{2}(\lambda(m+1)+\eta) \Gamma^{2}(m+2)} a_{m+1}^{2} .
\end{aligned}
$$

Therefore, we have

$$
\begin{aligned}
& a_{m+1}^{2}= \\
& \frac{\alpha^{2} \Gamma^{2}(\delta+\tau) \Gamma^{2}(\lambda(m+1)+\eta) \Gamma^{2}(m+2) \Gamma(\lambda(2 m+1)+\eta) \Gamma(2(m+1))\left(p_{2 m}+q_{2 m}\right)}{\alpha \Omega_{1}(\gamma, \lambda, \eta, \delta, \tau, m)+(1-\alpha) \Gamma(\lambda(2 m+1)+\eta) \Gamma(2(m+1)) \Omega_{2}^{2}(\gamma, \lambda, \eta, \delta, \tau, m)},
\end{aligned}
$$

where $\Omega_{1}(\gamma, \lambda, \eta, \delta, \tau, m)$ and $\Omega_{2}(\gamma, \lambda, \eta, \delta, \tau, m)$ are given by (9) and (10), respectively. 
Now, taking the absolute value of (21) and applying Lemma 1 for the coefficients $p_{2 m}$ and $q_{2 m}$, we deduce that

$$
\begin{aligned}
& \left|a_{m+1}\right| \leq \\
& \frac{2 \alpha \Gamma(\delta+\tau) \Gamma(\lambda(m+1)+\eta) \Gamma(m+2) \sqrt{\Gamma(\lambda(2 m+1)+\eta) \Gamma(2(m+1))}}{\sqrt{\left|\alpha \Omega_{1}(\gamma, \lambda, \eta, \delta, \tau, m)+(1-\alpha) \Gamma(\lambda(2 m+1)+\eta) \Gamma(2(m+1)) \Omega_{2}^{2}(\gamma, \lambda, \eta, \delta, \tau, m)\right|}} .
\end{aligned}
$$

This gives the desired estimate for $\left|a_{m+1}\right|$ as asserted in (7).

In order to find the bound on $\left|a_{2 m+1}\right|$, by subtracting (18) from (16), we get

$$
\begin{aligned}
& \frac{\Gamma(\delta+(2 m+1) \tau) \Gamma(\lambda+\eta)[\gamma(4 m(m+1)+2)+2 m(\gamma+1)+1]}{\Gamma(\delta+\tau) \Gamma(\lambda(2 m+1)+\eta) \Gamma(2(m+1))} \times \\
& \times\left(2 a_{2 m+1}-(m+1) a_{m+1}^{2}\right)=\alpha\left(p_{2 m}-q_{2 m}\right)+\frac{\alpha(\alpha-1)}{2}\left(p_{m}^{2}-q_{m}^{2}\right) .
\end{aligned}
$$

It follows from (19), (20) and (22) that

$$
\begin{aligned}
a_{2 m+1} & =\frac{\alpha^{2}(m+1) \Gamma^{2}(\delta+\tau) \Gamma^{2}(\lambda(m+1)+\eta) \Gamma^{2}(m+2)\left(p_{m}^{2}+q_{m}^{2}\right)}{4 \Omega_{2}^{2}(\gamma, \lambda, \eta, \delta, \tau, m)} \\
& +\frac{\alpha \Gamma(\delta+\tau) \Gamma(\lambda(2 m+1)+\eta) \Gamma(2(m+1))\left(p_{2 m}-q_{2 m}\right)}{2 \Gamma(\delta+(2 m+1) \tau) \Gamma(\lambda+\eta)[\gamma(4 m(m+1)+2)+2 m(\gamma+1)+1]} .
\end{aligned}
$$

Taking the absolute value of (23) and applying Lemma 1 once again for the coefficients $p_{m}, p_{2 m}, q_{m}$ and $q_{2 m}$, we obtain

$$
\begin{aligned}
\left|a_{2 m+1}\right| & \leq \frac{2 \alpha^{2}(m+1) \Gamma^{2}(\delta+\tau) \Gamma^{2}(\lambda(m+1)+\eta) \Gamma^{2}(m+2)}{\Omega_{2}^{2}(\gamma, \lambda, \eta, \delta, \tau, m)} \\
& +\frac{2 \alpha \Gamma(\delta+\tau) \Gamma(\lambda(2 m+1)+\eta) \Gamma(2(m+1))}{\Gamma(\delta+(2 m+1) \tau) \Gamma(\lambda+\eta)[\gamma(4 m(m+1)+2)+2 m(\gamma+1)+1]},
\end{aligned}
$$

which completes the proof of Theorem 1 .

Remark 2. In Theorem 1, if we choose $\gamma=\lambda=0$ and $\eta=\delta=\tau=1$, then we obtain the results which was given by Srivastava et al. [20, Theorem 2].

For one-fold symmetric bi-univalent functions, Theorem 1 reduces to the following corollary:

Corollary 1. Let $f \in \mathcal{R}_{\Sigma}(\gamma, \lambda, \eta, \delta, \tau ; \alpha)(0<\alpha \leq 1,0 \leq \gamma \leq 1)$ be given by (1). Then

$$
\left|a_{2}\right| \leq \frac{4 \alpha \Gamma(\delta+\tau) \Gamma(2 \lambda+\eta) \sqrt{6 \Gamma(3 \lambda+\eta)}}{\sqrt{\left|\alpha \Omega_{3}(\gamma, \lambda, \eta, \delta, \tau)+24(1-\alpha) \Gamma(3 \lambda+\eta) \Gamma^{2}(\delta+2 \tau) \Gamma^{2}(\lambda+\eta)(3 \gamma+1)^{2}\right|}}
$$

and

$$
\left|a_{3}\right| \leq \frac{4 \alpha^{2} \Gamma^{2}(\delta+\tau) \Gamma^{2}(2 \lambda+\eta)}{\Gamma^{2}(\delta+2 \tau) \Gamma^{2}(\lambda+\eta)(3 \gamma+1)^{2}}+\frac{4 \alpha \Gamma(\delta+\tau) \Gamma(3 \lambda+\eta)}{\Gamma(\delta+3 \tau) \Gamma(\lambda+\eta)(4 \gamma+1)},
$$

where

$$
\Omega_{3}(\gamma, \lambda, \eta, \delta, \tau)=24 \Gamma(\delta+\tau) \Gamma^{2}(2 \lambda+\eta) \Gamma(\delta+3 \tau) \Gamma(\lambda+\eta)(4 \gamma+1) .
$$


Remark 3. In Corollary 1, if we choose $\gamma=\lambda=0$ and $\eta=\delta=\tau=1$, then we obtain the results which was given by Srivastava et al. [18, Theorem 1].

3. Coefficient estimates for the functions Class

$$
\mathcal{R}_{\Sigma_{m}}^{*}(\gamma, \lambda, \eta, \delta, \tau ; \beta)
$$

Definition 2. For $0 \leq \beta<1,0 \leq \gamma \leq 1$ and $m \in \mathbb{N}$, a function $f \in \Sigma_{m}$ given by (3) is said to be in the class $\mathcal{R}_{\Sigma_{m}}^{*}(\gamma, \lambda, \eta, \delta, \tau ; \beta)$ if it satisfies the following conditions:

$$
\operatorname{Re}\left\{\gamma z\left(\mathcal{H}_{\lambda, \eta}^{\delta, \tau} f(z)\right)^{\prime \prime}+(2 \gamma+1)\left(\mathcal{H}_{\lambda, \eta}^{\delta, \tau} f(z)\right)^{\prime}-2 \gamma\right\}>\beta
$$

and

$$
\operatorname{Re}\left\{\gamma w\left(\mathcal{H}_{\lambda, \eta}^{\delta, \tau} g(w)\right)^{\prime \prime}+(2 \gamma+1)\left(\mathcal{H}_{\lambda, \eta}^{\delta, \tau} g(w)\right)^{\prime}-2 \gamma\right\}>\beta,
$$

where the function $g=f^{-1}$ is given by (4).

In particular, for one-fold symmetric bi-univalent functions, we denote the class

$$
\mathcal{R}_{\Sigma_{1}}^{*}(\gamma, \lambda, \eta, \delta, \tau ; \beta) \equiv \mathcal{R}_{\Sigma}^{*}(\gamma, \lambda, \eta, \delta, \tau ; \beta) .
$$

Remark 4. If we put $\gamma=\lambda=0$ and $\eta=\delta=\tau=1$ :

(1) The class $\mathcal{R}_{\Sigma_{m}}^{*}(\gamma, \lambda, \eta, \delta, \tau ; \beta)$ reduces to the class $\mathcal{H}_{\Sigma, m}(\beta)$ which was given by Srivastava et al. [20];

(2) The class $\mathcal{R}_{\Sigma}^{*}(\gamma, \lambda, \eta, \delta, \tau ; \beta)$ reduces to the class $\mathcal{H}_{\Sigma}(\beta)$ which was given by Srivastava et al. [18].

Theorem 2. Let $f \in \mathcal{R}_{\Sigma_{m}}^{*}(\gamma, \lambda, \eta, \delta, \tau ; \beta) \quad(0 \leq \beta<1,0 \leq \gamma \leq 1, m \in \mathbb{N})$ be given by (3). Then

$$
\begin{aligned}
& \left|a_{m+1}\right| \\
& \leq 2 \sqrt{\frac{\Gamma(\delta+\tau) \Gamma(\lambda(2 m+1)+\eta) \Gamma(2(m+1))(1-\beta)}{(m+1) \Gamma(\delta+(2 m+1) \tau) \Gamma(\lambda+\eta)[\gamma(4 m(m+1)+2)+2 m(\gamma+1)+1]}}
\end{aligned}
$$

and

$$
\begin{aligned}
\left|a_{2 m+1}\right| & \leq \frac{2 \Gamma^{2}(\delta+\tau) \Gamma^{2}(\lambda(m+1)+\eta) \Gamma^{2}(m+2)(1-\beta)^{2}(m+1)}{\Omega_{2}^{2}(\gamma, \lambda, \eta, \delta, \tau, m)} \\
& +\frac{2 \Gamma(\delta+\tau) \Gamma(\lambda(2 m+1)+\eta) \Gamma(2(m+1))(1-\beta)}{\Gamma(\delta+(2 m+1) \tau) \Gamma(\lambda+\eta)[\gamma(4 m(m+1)+2)+2 m(\gamma+1)+1]},
\end{aligned}
$$

where $\Omega_{2}(\gamma, \lambda, \eta, \delta, \tau, m)$ is given by (10).

Proof. It follows from conditions (24) and (25) that there exist $p, q \in \mathcal{P}$ such that

$$
\gamma z\left(\mathcal{H}_{\lambda, \eta}^{\delta, \tau} f(z)\right)^{\prime \prime}+(2 \gamma+1)\left(\mathcal{H}_{\lambda, \eta}^{\delta, \tau} f(z)\right)^{\prime}-2 \gamma=\beta+(1-\beta) p(z)
$$


and

(29) $\gamma w\left(\mathcal{H}_{\lambda, \eta}^{\delta, \tau} g(w)\right)^{\prime \prime}+(2 \gamma+1)\left(\mathcal{H}_{\lambda, \eta}^{\delta, \tau} g(w)\right)^{\prime}-2 \gamma=\beta+(1-\beta) q(w)$,

where $p(z)$ and $q(w)$ have the forms (13) and (14), respectively. Equating coefficients (28) and (29) yields

$$
\begin{aligned}
& \frac{\Gamma(\delta+(m+1) \tau) \Gamma(\lambda+\eta)\left[\gamma\left((m+1)^{2}+1\right)+m(\gamma+1)+1\right]}{\Gamma(\delta+\tau) \Gamma(\lambda(m+1)+\eta) \Gamma(m+2)} a_{m+1} \\
& =(1-\beta) p_{m},
\end{aligned}
$$

$$
\begin{aligned}
& \frac{\Gamma(\delta+(2 m+1) \tau) \Gamma(\lambda+\eta)[\gamma(4 m(m+1)+2)+2 m(\gamma+1)+1]}{\Gamma(\delta+\tau) \Gamma(\lambda(2 m+1)+\eta) \Gamma(2(m+1))} a_{2 m+1} \\
& =(1-\beta) p_{2 m}
\end{aligned}
$$

$$
\begin{aligned}
& -\frac{\Gamma(\delta+(m+1) \tau) \Gamma(\lambda+\eta)\left[\gamma\left((m+1)^{2}+1\right)+m(\gamma+1)+1\right]}{\Gamma(\delta+\tau) \Gamma(\lambda(m+1)+\eta) \Gamma(m+2)} a_{m+1} \\
& =(1-\beta) q_{m}
\end{aligned}
$$

and

$$
\begin{aligned}
& \frac{\Gamma(\delta+(2 m+1) \tau) \Gamma(\lambda+\eta)[\gamma(4 m(m+1)+2)+2 m(\gamma+1)+1]}{\Gamma(\delta+\tau) \Gamma(\lambda(2 m+1)+\eta) \Gamma(2(m+1))} \times \\
& \times\left((m+1) a_{m+1}^{2}-a_{2 m+1}\right)=(1-\beta) q_{2 m} .
\end{aligned}
$$

From (30) and (32), we get

$$
p_{m}=-q_{m}
$$

and

$$
\begin{aligned}
& \frac{2 \Gamma^{2}(\delta+(m+1) \tau) \Gamma^{2}(\lambda+\eta)\left[\gamma\left((m+1)^{2}+1\right)+m(\gamma+1)+1\right]^{2}}{\Gamma^{2}(\delta+\tau) \Gamma^{2}(\lambda(m+1)+\eta) \Gamma^{2}(m+2)} a_{m+1}^{2} \\
& =(1-\beta)^{2}\left(p_{m}^{2}+q_{m}^{2}\right) .
\end{aligned}
$$

Adding (31) and (33), we obtain

$$
\begin{aligned}
& \frac{(m+1) \Gamma(\delta+(2 m+1) \tau) \Gamma(\lambda+\eta)[\gamma(4 m(m+1)+2)+2 m(\gamma+1)+1]}{\Gamma(\delta+\tau) \Gamma(\lambda(2 m+1)+\eta) \Gamma(2(m+1))} \times \\
& \times a_{m+1}^{2}=(1-\beta)\left(p_{2 m}+q_{2 m}\right) .
\end{aligned}
$$

Therefore, we have

$$
a_{m+1}^{2}=\frac{\Gamma(\delta+\tau) \Gamma(\lambda(2 m+1)+\eta) \Gamma(2(m+1))(1-\beta)\left(p_{2 m}+q_{2 m}\right)}{(m+1) \Gamma(\delta+(2 m+1) \tau) \Gamma(\lambda+\eta)[\gamma(4 m(m+1)+2)+2 m(\gamma+1)+1]} .
$$

Applying Lemma 1 for the coefficients $p_{2 m}$ and $q_{2 m}$, we obtain

$$
\left|a_{m+1}\right| \leq 2 \sqrt{\frac{\Gamma(\delta+\tau) \Gamma(\lambda(2 m+1)+\eta) \Gamma(2(m+1))(1-\beta)}{(m+1) \Gamma(\delta+(2 m+1) \tau) \Gamma(\lambda+\eta)[\gamma(4 m(m+1)+2)+2 m(\gamma+1)+1]}} .
$$


This gives the desired estimate for $\left|a_{m+1}\right|$ as asserted in (26).

In order to find the bound on $\left|a_{2 m+1}\right|$, by subtracting (33) from (31), we get

$$
\begin{aligned}
& \frac{\Gamma(\delta+(2 m+1) \tau) \Gamma(\lambda+\eta)[\gamma(4 m(m+1)+2)+2 m(\gamma+1)+1]}{\Gamma(\delta+\tau) \Gamma(\lambda(2 m+1)+\eta) \Gamma(2(m+1))} \times \\
& \times\left(2 a_{2 m+1}-(m+1) a_{m+1}^{2}\right)=(1-\beta)\left(p_{2 m}-q_{2 m}\right),
\end{aligned}
$$

or equivalently

$$
\begin{aligned}
a_{2 m+1} & =\frac{m+1}{2} a_{m+1}^{2} \\
& +\frac{\Gamma(\delta+\tau) \Gamma(\lambda(2 m+1)+\eta) \Gamma(2(m+1))(1-\beta)\left(p_{2 m}-q_{2 m}\right)}{2 \Gamma(\delta+(2 m+1) \tau) \Gamma(\lambda+\eta)[\gamma(4 m(m+1)+2)+2 m(\gamma+1)+1]} .
\end{aligned}
$$

Upon substituting the value of $a_{m+1}^{2}$ from (35), it follows that

$$
\begin{aligned}
a_{2 m+1} & =\frac{\Gamma^{2}(\delta+\tau) \Gamma^{2}(\lambda(m+1)+\eta) \Gamma^{2}(m+2)(1-\beta)^{2}(m+1)\left(p_{m}^{2}+q_{m}^{2}\right)}{4 \Omega_{2}^{2}(\gamma, \lambda, \eta, \delta, \tau, m)} \\
& +\frac{\Gamma(\delta+\tau) \Gamma(\lambda(2 m+1)+\eta) \Gamma(2(m+1))(1-\beta)\left(p_{2 m}-q_{2 m}\right)}{2 \Gamma(\delta+(2 m+1) \tau) \Gamma(\lambda+\eta)[\gamma(4 m(m+1)+2)+2 m(\gamma+1)+1]},
\end{aligned}
$$

where $\Omega_{2}(\gamma, \lambda, \eta, \delta, \tau, m)$ is given by (10).

Applying Lemma 1 once again for the coefficients $p_{m}, p_{2 m}, q_{m}$ and $q_{2 m}$, we obtain

$$
\begin{aligned}
\left|a_{2 m+1}\right| & \leq \frac{2 \Gamma^{2}(\delta+\tau) \Gamma^{2}(\lambda(m+1)+\eta) \Gamma^{2}(m+2)(1-\beta)^{2}(m+1)}{\Omega_{2}^{2}(\gamma, \lambda, \eta, \delta, \tau, m)} \\
& +\frac{2 \Gamma(\delta+\tau) \Gamma(\lambda(2 m+1)+\eta) \Gamma(2(m+1))(1-\beta)}{\Gamma(\delta+(2 m+1) \tau) \Gamma(\lambda+\eta)[\gamma(4 m(m+1)+2)+2 m(\gamma+1)+1]},
\end{aligned}
$$

which completes the proof of Theorem 2 .

Remark 5. In Theorem 2, if we choose $\gamma=\lambda=0$ and $\eta=\delta=\tau=1$, then we obtain the results which was given by Srivastava et al. [20, Theorem 3].

For one-fold symmetric bi-univalent functions, Theorem 2 reduces to the following corollary:

Corollary 2. Let $f \in \mathcal{R}_{\Sigma}^{*}(\gamma, \lambda, \eta, \delta, \tau ; \beta)(0 \leq \beta<1,0 \leq \gamma \leq 1)$ be given by (1). Then

$$
\left|a_{2}\right| \leq 2 \sqrt{\frac{\Gamma(\delta+\tau) \Gamma(3 \lambda+\eta)(1-\beta)}{\Gamma(\delta+3 \tau) \Gamma(\lambda+\eta)(4 \gamma+1)}}
$$

and

$$
\left|a_{3}\right| \leq \frac{8 \Gamma^{2}(\delta+\tau) \Gamma^{2}(2 \lambda+\eta)(1-\beta)^{2}}{\Gamma^{2}(\delta+2 \tau) \Gamma^{2}(\lambda+\eta)(3 \gamma+1)^{2}}+\frac{4 \Gamma(\delta+\tau) \Gamma(3 \lambda+\eta)(1-\beta)}{\Gamma(\delta+3 \tau) \Gamma(\lambda+\eta)(4 \gamma+1)} .
$$

Remark 6. In Corollary 2, if we choose $\gamma=\lambda=0$ and $\eta=\delta=\tau=1$, then we obtain the results which was given by Srivastava et al. [18, Theorem 2]. 


\section{Conclusion}

In the present work, we have introduced two new classes of analytic and $m$-fold symmetric bi-univalent functions in the open unit disk $U$ associated with Mittag-Leffler function. We have then derived the initial coefficient estimations for functions belonging to these new classes. Further by specializing the parameters, several consequences of these new classes are mentioned.

\section{REFERENCES}

[1] E. A. Adegani, S. Bulut, A. A. Zireh, Coefficient estimates for a subclass of analytic bi-univalent functions, Bulletin of the Korean Mathematical Society, 55 (2) (2018), 405-413.

[2] Ş. Altınkaya, S. Yalçin, On some subclasses of $m$-fold symmetric bi-univalent functions, Communications Faculty of Sciences University of Ankara Series A1, 67(1) (2018), 29-36.

[3] A. A. Attiya, Some applications of Mittag-Leffler function in the unit disk, Filomat, 30 (7) (2016), 2075-2081.

[4] M. Caglar, E. Deniz, H. M. Srivastava, Second Hankel determinant for certain subclasses of bi-univalent functions, Turkish Journal of Mathematics, 41 (2017), 694-706.

[5] P. L. Duren, Univalent Functions, Grundlehren der Mathematischen Wissenschaften, Band 259, Springer Verlag, New York, Berlin, Heidelberg and Tokyo, 1983.

[6] S. S. Eker, Coefficient bounds for subclasses of $m$-fold symmetric bi-univalent functions, Turkish Journal of Mathematics, 40 (2016), 641-646.

[7] W. Koepf, Coefficients of symmetric functions of bounded boundary rotations, Proceedings of the American Mathematical Society, 105 (1989), 324-329.

[8] N. Magesh, S. Bulut, Chebyshev polynomial coefficient estimates for a class of analytic bi-univalent functions related to pseudo-starlike functions, Afrika Matematika, 29 (1-2) (2018), 203-209.

[9] G. M. Mittag-Leffler, Sur la nouvelle function, Comptes Rendus de l'Academie des Sciences Paris, 137 (1903), 554-558.

[10] G. M. Mittag-Leffler, Sur la representation analytique d'une function monogene (cinquieme note), Acta Mathematica, 29 (1905), 101-181.

[11] G. Murugusundaramoorthy, C. Selvaraj, O. S. Babu, Coefficient estimates for Pascutype subclasses of bi-univalent functions based on subordination, International Journal of Nonliner Science, 19 (1) (2015), 47-52.

[12] H. M. Srivastava, Operators of basic (or q-) calculus and fractional q-calculus and their applications in geometric function theory of complex analysis, Iranian Journal of Science and Technology, Transactions A: Science, 44 (2020), 327-344.

[13] H. M. Srivastava, Ş. Altınkaya, S. Yalçin, Certain subclasses of bi-univalent functions associated with the Horadam polynomials, Iranian Journal of Science and Technology, Transactions A: Science, 43 (2019), 1873-1879.

[14] H. M. Srivastava, S. S. Eker, S. G. Hamidi, J. M. Jahangiri, Faber polynomial coeffcient estimates for bi-univalent functions defined by the Tremblay fractional derivative operator, Bulletin of the Iranian Mathematical Society, 44 (1) (2018), 149-157.

[15] H. M. Srivastava, S. Gaboury, F. Ghanim, Coefficient estimates for some subclasses of $m$-fold symmetric bi-univalent functions, Acta Universitatis Apulensis Mathematics Informatics, 41 (2015), 153-164. 
[16] H. M. Srivastava, S. Gaboury, F. Ghanim, Initial coefficient estimates for some subclasses of $m$-fold symmetric bi-univalent functions, Acta Mathematica Scientia Series B English Edition, 36 (2016), 863-871.

[17] H. M. Srivastava, S. Gaboury, F. Ghanim, Coefficient estimates for some general subclasses of analytic and bi-univalent functions, Afrika Matematika, 28 (2017), 693-706.

[18] H. M. Srivastava, A. K. Mishra, P. Gochhayat, Certain subclasses of analytic and bi-univalent functions, Applied Mathematics Letters, 23 (2010), 1188-1192.

[19] H. M. Srivastava, A. Motamednezhad, E. A. Adegani, Faber polynomial coefficient estimates for bi-univalent functions defined by using differential subordination and a certain fractional derivative operator, Mathematics, 8 (2020), Article ID: 172, 1-12.

[20] H. M. Srivastava, S. Sivasubramanian, R. Sivakumar, Initial coefficient bounds for a subclass of $m$-fold symmetric bi-univalent functions, Tbilisi Mathematical Journal, 7 (2) (2014), 1-10.

[21] H. M. Srivastava, Z. Tomovski, Fractional calculus with an integral operator containing a generalized Mittag-Leffler function in the kernal, Applied Mathematics and Computation, 211 (2009), 198-210.

[22] H. M. Srivastava, A. K. Wanas, Initial Maclaurin coefficient bounds for new subclasses of analytic and $m$-fold symmetric bi-univalent functions defined by a linear combination, Kyungpook Mathematical Journal, 59 (3) (2019), 493-503.

[23] H. M. Srivastava, A. Zireh, S. Hajiparvaneh, Coefficient estimates for some subclasses of m-fold symmetric bi-univalent functions, Filomat, 32 (2018), 3143-3153.

[24] H. Tang, H. M. Srivastava, S. Sivasubramanian, P. Gurusamy, The Fekete-Szegö functional problems for some subclasses of $m$-fold symmetric bi-univalent functions, Journal of Mathematical Inequalities, 10 (2016), 1063-1092.

[25] A. K. Wanas, A. L. Alina, Applications of Horadam polynomials on Bazilevič biunivalent function satisfying subordinate conditions, Journal of Physics: Conference Series, 1294 (2019), 1-6.

[26] A. K. Wanas, A. H. Majeed, Certain new subclasses of analytic and $m$-fold symmetric bi-univalent functions, Applied Mathematics E-Notes, 18 (2018), 178-188.

[27] A. K. Wanas, S. Yalçin, Initial coefficient estimates for a new subclasses of analytic and $m$-Fold symmetric bi-univalent functions, Malaya Journal of Matematik, 7 (3) (2019), 472-476.

\author{
Abbas Kareem Wanas \\ Department of Mathematics \\ College of Science \\ UNIVERSITY OF AL-QADISIYAH \\ IRAQ \\ E-mail address: abbas.kareem.w@qu.edu.iq
}

\section{Huo TANG}

School of Mathematics and Statistics

Chifeng University

Chifeng 024000, Inner Mongolia

China

E-mail address: thth2009@163.com 